\title{
Flexible affine cones over del Pezzo surfaces of degree 4
}

\author{
Jihun Park ${ }^{1,2}$ • Joonyeong Won ${ }^{3}$
}

Received: 22 December 2014 / Revised: 6 May 2015 / Accepted: 28 May 2015 /

Published online: 19 June 2015

(C) Springer International Publishing AG 2015

Abstract For an arbitrary ample divisor $A$ on a smooth del Pezzo surface $S$ of degree 4 , we show that the affine cone of $S$ defined by $A$ is flexible.

Keywords Affine cone - Ample divisor · Cylinder · del Pezzo surface · Infinitely transitive action $\cdot$ Flexible variety

Mathematics Subject Classification $14 \mathrm{~J} 26 \cdot 14 \mathrm{R} 20 \cdot 14 \mathrm{R} 25$

\section{Introduction}

All considered varieties are assumed to be algebraic and defined over an algebraically closed field of characteristic 0 throughout this article.

The first author has been supported by IBS-R003-G2, Institute for Basic Science in Korea and the second author has been supported by the National Research Foundation in Korea (NRF-2014R1A1A2056432).

$\triangle$ Jihun Park

wlog@postech.ac.kr

Joonyeong Won

leonwon@kias.re.kr

1 Center for Geometry and Physics, Institute for Basic Science (IBS), 77 Cheongam-ro, Nam-gu, Pohang, Gyeongbuk 790-784, Korea

2 Department of Mathematics, POSTECH, 77 Cheongam-ro, Nam-gu, Pohang, Gyeongbuk 790-784, Korea

3 Algebraic Structure and its Applications Research Center, KAIST, 335 Gwahangno, Yuseong-gu, Daejeon 305-701, Korea 
For a positive integer $m$, a group $G$ is said to act $m$-transitively on a set $X$ if the action is transitive on $m$-tuples of pairwise distinct points of $X$. Furthermore, an action is called infinitely transitive on $X$ if it is $m$-transitive for each positive integer $m$.

For an algebraic variety $X$, the subgroup of $\operatorname{Aut}(X)$ generated by all algebraic oneparameter unipotent subgroups of $\operatorname{Aut}(X)$ is denoted by $\operatorname{SAut}(X)$. The group $\operatorname{SAut}(X)$ is called the special automorphism group of $X$. Meanwhile, an algebraic variety $X$ is called flexible if the tangent space of $X$ at each smooth point $x \in X$ is spanned by the tangent vectors to the orbits $H \cdot x$ of one-parameter unipotent subgroups $H$ of $\operatorname{Aut}(X)$.

The following theorem from [1] shows the connection between flexibility and infinite transitivity.

Theorem 1.1 Let $X$ be an irreducible affine algebraic variety of dimension at least 2. Then the following are equivalent:

- $X$ is flexible;

- $\operatorname{SAut}(X)$ acts transitively on the smooth locus of $X$;

- SAut $(X)$ acts infinitely transitively on the smooth locus of X.

In addition, [1] proves that every flexible variety is unirational. On the other hand, in [4], it is conjectured that every unirational variety is stably birational to an infinitely transitive variety and it is proved in some cases. Kaliman and Zaidenberg proved that every hypersurface in $\mathbb{A}^{n+2}$ defined by an equation $u v=f\left(x_{1}, \ldots, x_{n}\right)$ for a nonconstant polynomial $f$ has the infinitely transitive property [8]. Moreover, in [3], it is proved that the suspensions over flexible varieties are also flexible. One can find some other examples of flexible varieties in [1,2].

In the present paper we consider affine cones over smooth del Pezzo surfaces polarized by arbitrary ample divisors. For smooth del Pezzo surfaces of degrees less than or equal to 3 , the non-existence of $\mathbb{G}_{\mathrm{a}}$-actions on affine cones by their anticanonical divisors was proved in $[5,10]$. In [6], the existence and non-existence of $\mathbb{G}_{\mathrm{a}}$-actions on affine cones over anticanonically polarized del Pezzo surfaces with du Val singularities were fully established according to their singularities and degrees.

Since smooth del Pezzo surfaces of degrees greater than or equal to 6 are toric, affine cones over such surfaces are flexible by [3]. In [11], it is also shown that affine cones over the smooth del Pezzo surface of degree 5 polarized by arbitrary ample divisors are flexible.

Theorem 1.2 Let $S$ be a smooth del Pezzo surface of degree at least 5. For every ample divisor $H$, the affine cone

$$
\operatorname{Affcone}_{H}(S)=\operatorname{Spec} \bigoplus_{m=0}^{\infty} H^{0}\left(S, \mathcal{O}_{S}(m H)\right)
$$

is flexible.

In the case of degree 4 the paper [11] proves the flexibility for certain ample divisors including anticanonical divisor. In order to complete the case of degree 4 , we prove the following 
Main Theorem Let $S$ be a smooth del Pezzo surface of degree 4. For an arbitrary ample divisor $H$ on $S$, the affine cone $\operatorname{Affcone}_{H}(S)$ is flexible.

\section{Cylinder, $\mathbb{G}_{\mathbf{a}}$-action and flexibility}

Let $Y$ be a projective variety and $H$ be an ample divisor on $Y$. The following concepts play central role in the study of the flexibility of affine cones over varieties polarized by ample divisors.

Definition 2.1 An open subset $U$ of $Y$ is called a cylinder if $U$ is isomorphic to $Z \times \mathbb{A}^{1}$ for some affine variety $Z$. A cylinder $U$ is called $H$-polar if the complement of $U$ is the support of an effective $\mathbb{Q}$-divisor that is $\mathbb{Q}$-linearly equivalent to $H$.

Definition 2.2 A subset $W$ of $Y$ is said to be invariant with respect to a cylinder $U=Z \times \mathbb{A}^{1}$ if $W \cap U=\pi^{-1}(\pi(W))$, where $\pi: U \rightarrow Z$ is the projection on the first factor.

Definition 2.3 The variety $Y$ is said to be transversally covered by cylinders $U_{i}, i \in I$, if

- $Y=\bigcup_{i \in I} U_{i}$

- there is no proper non-empty subset of $Y$ invariant with respect to all $U_{i}$.

Let $H$ be an ample divisor on a smooth projective variety $Y$. Put

$$
\operatorname{Affcone}_{H}(Y)=\operatorname{Spec} \bigoplus_{m=0}^{\infty} H^{0}\left(Y, \mathcal{O}_{Y}(m H)\right) \text {. }
$$

The following two theorems show how the concepts above engage in the study of flexibility of the affine cone $\operatorname{Affcone}_{H}(Y)$.

Theorem 2.4 ([9, Corollary 2.12]) The affine cone Affcone $_{H}(Y)$ admits an effective $\mathbb{G}_{\mathrm{a}}$-action if and only if $Y$ contains an $H$-polar cylinder.

Theorem 2.5 ([11, Theorem 5]) If Y has a transversal covering by H-polar cylinders, then the affine cone $\operatorname{Affcone}_{H}(Y)$ is flexible.

Before we proceed, we present four basic cylinders on $\mathbb{P}^{2}$ and $\mathbb{P}^{1} \times \mathbb{P}^{1}$ that will be used in our constructions of transversal coverings.

Example 2.6 Let $L_{1}, L_{2}, L_{3}$ be three lines on $\mathbb{P}^{2}$ meeting at a single point. Then $\mathbb{P}^{2} \backslash\left(L_{1} \cup L_{2} \cup L_{3}\right)$ is a cylinder that is isomorphic to an $\mathbb{A}^{1}$-bundle over a two-pointdeleted affine line $\mathbb{A}_{* *}^{1}$.

Example 2.7 Let $C$ be an irreducible conic on $\mathbb{P}^{2}$ and let $L$ be a line tangent to $C$. The divisor $C+L$ defines a cylinder isomorphic to an $\mathbb{A}^{1}$-bundle over a one-point-deleted affine line $\mathbb{A}_{*}^{1}$, i.e.,

$$
\mathbb{P}^{2} \backslash(C \cup L) \cong \mathbb{A}_{*}^{1} \times \mathbb{A}^{1}
$$


Example 2.8 Let $C$ be a cuspidal cubic with a cusp at a point $P$ on $\mathbb{P}^{2}$ and let $T$ be the Zariski tangent line to $C$ at the point $P$. Then $\mathbb{P}^{2} \backslash(C \cup T)$ is isomorphic to $\mathbb{A}_{*}^{1} \times \mathbb{A}^{1}$.

Example 2.9 Let $C$ be an irreducible curve of bidegree $(1,2)$ on $\mathbb{P}^{1} \times \mathbb{P}^{1}$. There is a curve $L$ of bidegree $(1,0)$ tangent to the curve $C$. Let $P$ be the intersection point of $C$ and $L$ and let $H$ be the curve of bidegree $(0,1)$ that passes through the point $P$. Then the divisor $C+L+H$ defines a cylinder on $\mathbb{P}^{1} \times \mathbb{P}^{1}$ that is isomorphic to $\mathbb{A}_{*}^{1} \times \mathbb{A}^{1}$. To see this, we take the blow up $\rho: S_{7} \rightarrow \mathbb{P}^{1} \times \mathbb{P}^{1}$ at the point $P$. Let $E$ be the exceptional curve of $\rho$ and $\widetilde{C}$ be the proper transform of $C$ by $\rho$. The proper transforms $\widetilde{H}, \widetilde{L}$ of $H$ and $L$ by $\rho$ are disjoint $(-1)$-curves on $S_{7}$. By contracting these two $(-1)$-curves, we obtain a contraction $\psi: S_{7} \rightarrow \mathbb{P}^{2}$. The curve $\psi(\widetilde{C})$ is an irreducible conic and $\psi(E)$ is a line tangent to $\psi(\widetilde{C})$. Therefore, $C+L+H$ defines a cylinder since

$$
\begin{aligned}
\mathbb{P}^{1} \times \mathbb{P}^{1} \backslash(C \cup L \cup H) & \cong S_{7} \backslash(\widetilde{C} \cup \widetilde{L} \cup \widetilde{H} \cup E) \\
& \cong \mathbb{P}^{2} \backslash(\psi(\widetilde{C}) \cup \psi(E)) \cong \mathbb{A}_{*}^{1} \times \mathbb{A}^{1} .
\end{aligned}
$$

\section{Ample divisors on smooth del Pezzo surfaces of degree 4}

Let $S$ be a smooth del Pezzo surface of degree 4 . It can be obtained by blowing up $\mathbb{P}^{2}$ at five points in general position. Let $\phi: S \rightarrow \mathbb{P}^{2}$ be such a blow up and $E_{1}, \ldots, E_{5}$ be its exceptional curves. Denote the point $\phi\left(E_{i}\right)$ by $P_{i}$.

Let $h$ be the divisor class of $S$ corresponding to $\phi^{*}\left(\mathcal{O}_{\mathbb{P}^{2}}(1)\right)$ and $e_{i}$ be the class of the exceptional curves $E_{i}$, where $i=1, \ldots, 5$. Since the classes $h, e_{1}, \ldots, e_{5}$ form an orthogonal basis of the Picard group of $S$, for a divisor $A$ on $S$ we may write $[A]=\beta h+\sum_{i=1}^{5} \beta_{i} e_{i}$, where $\beta$ and $\beta_{i}$ are constants. It is well known that the divisor $A$ is ample if and only if the following inequalities hold

$$
\begin{array}{ll}
\beta \geq-\beta_{i}>0 & \text { for } i=1,2,3,4,5 \\
\beta+\beta_{i}+\beta_{j}>0 & \text { for } i \neq j \\
2 \beta+\beta_{1}+\beta_{2}+\beta_{3}+\beta_{4}+\beta_{5}>0 . &
\end{array}
$$

In other words, these relations define the ample cone of $S$. The Mori cone $\overline{\mathbb{N E}}(S)$ of the surface $S$, the dual of the closure of the ample cone, is polyhedral. Moreover, it is generated by all (-1)-curves on $S$ [7, Theorem 8.2.23].

From now on, the divisor $A$ is always assumed to be ample, unless otherwise stated. The following method to express the divisor $A$ in terms of $-K_{S}$ and (-1)-curves is adopted from an ongoing joint work of the authors with Cheltsov.

For the log pair $(S, A)$, we define an invariant of $(S, A)$ by

$$
\mu=\inf \left\{\lambda \in \mathbb{Q}_{>0}: \text { the } \mathbb{Q} \text {-divisor } K_{S}+\lambda A \text { is pseudo-effective }\right\} .
$$

The invariant $\mu$ is always attained by a positive rational number. There is the smallest face $\Delta_{(S, A)}$ of the boundary of the Mori cone $\overline{\mathbb{N E}}(S)$ that contains $K_{S}+\mu A$.

Let $\phi: S \rightarrow Z$ be the contraction given by the face $\Delta_{(S, A)}$. Then either $\phi$ is a birational morphism or a conic bundle with $Z \cong \mathbb{P}^{1}[7,8.2 .6]$. In the former case 
$\Delta_{(S, A)}$ is generated by $r$ disjoint $(-1)$-curves contracted by $\phi$, where $r \leq 5$. In the later case $\Delta_{(S, A)}$ is generated by the $(-1)$-curves in the four reducible fibers of $\phi$. Each reducible fiber consists of two $(-1)$-curves that intersect transversally at one point.

Suppose that $\phi$ is birational. Let $E_{1}, \ldots, E_{r}$ be all $(-1)$-curves contained in $\Delta_{(S, A)}$. These are disjoint and generate the face $\Delta_{(S, A)}$. Therefore,

$$
K_{S}+\mu A \sim_{\mathbb{Q}} \sum_{i=1}^{r} a_{i} E_{i}
$$

for some positive rational numbers $a_{1}, \ldots, a_{r}$ [7, 8.2.6]. We have $a_{i}<1$ for every $i$ because $A \cdot E_{i}>0$. Vice versa, for every positive rational numbers $a_{1}, \ldots, a_{r}<1$, the divisor

$$
-K_{S}+\sum_{i=1}^{r} a_{i} E_{i}
$$

is ample.

Suppose that $\phi$ is a conic bundle. Then there are a 0 -curve $B$ and four disjoint $(-1)$-curves $E_{1}, E_{2}, E_{3}, E_{4}$, each of which is contained in a distinct fiber of $\phi$, such that

$$
K_{S}+\mu A \sim_{\mathbb{Q}} a B+\sum_{i=1}^{4} a_{i} E_{i}
$$

for some positive rational number $a$ and non-negative rational numbers $a_{1}, a_{2}, a_{3}, a_{4}<$ $1[7,8.2 .6]$. In particular, these curves generate the face $\Delta_{(S, A)}$. Vice versa, for every positive rational number $a$ and non-negative rational numbers $a_{1}, a_{2}, a_{3}, a_{4}<1$ the divisor

$$
-K_{S}+a B+\sum_{i=1}^{r} a_{i} E_{i}
$$

is ample.

\section{Proof of Main Theorem}

As before, let $S$ be a smooth del Pezzo surface of degree 4 and $A$ be an ample divisor on $S$. For the given log pair $(S, A)$, the contraction of the face $\Delta_{(S, A)}$ is either a birational morphism or a conic bundle. We prove that $S$ has transversal coverings by $A$-polar cylinders in both the cases. We may assume that $\Delta_{(S, A)}$ is a positive dimensional face since we already know that the affine cone over the polarization $\left(S,-n K_{S}\right)$ for every $n \geq 1$ is flexible [11]. 


\subsection{Birational morphism case}

We suppose that the contraction $\phi: S \rightarrow Z$ by the face $\Delta_{(S, A)}$ is birational. There are $r$ disjoint $(-1)$-curves $E_{1}, \ldots, E_{r}$ that generate the face $\Delta_{(S, A)}$, where $1 \leq r \leq 5$. In addition, we can find $5-r$ disjoint $(-1)$-curves $E_{r+1}, \ldots, E_{5}$ on $S$ that intersect none of the (-1)-curves $E_{1}, \ldots, E_{r}$. We are then able to obtain a birational morphism $\pi: S \rightarrow \mathbb{P}^{2}$ by contracting the five disjoint $(-1)$-curves $E_{1}, \ldots, E_{5}$ on $S$ to $\mathbb{P}^{2}$. Furthermore, we may write

$$
K_{S}+\mu A \sim_{\mathbb{Q}} \sum_{i=1}^{5} a_{i} E_{i},
$$

where $a_{i}$ are rational numbers with $0<a_{i}<1$ for $i=1, \ldots, r$ and $a_{i}=0$ for $i=r+1, \ldots, 5$.

Denote $\pi\left(E_{i}\right)$ by $P_{i}$. Let $L_{i j}$ be the line determined by the points $P_{i}$ and $P_{j}$ on $\mathbb{P}^{2}$ and $\widetilde{L}_{i j}$ be its proper transform by the morphism $\pi$. Consider the three intersection points

$$
Q_{1}=L_{23} \cap L_{45}, \quad Q_{2}=L_{24} \cap L_{35}, \quad Q_{3}=L_{25} \cap L_{34} .
$$

We then denote the line determined by the points $P_{1}$ and $Q_{i}$ by $L_{i}$ and its proper transform on $S$ by $\widetilde{L}_{i}$. Note that it is possible for two of the lines $L_{1}, L_{2}, L_{3}$ to coincide, but not for three of them.

Consider the sets

$$
\begin{aligned}
& U_{1}=\pi^{-1}\left(\mathbb{P}^{2} \backslash\left(L_{23} \cup L_{45} \cup L_{1}\right)\right), \\
& U_{2}=\pi^{-1}\left(\mathbb{P}^{2} \backslash\left(L_{24} \cup L_{35} \cup L_{2}\right)\right), \\
& U_{3}=\pi^{-1}\left(\mathbb{P}^{2} \backslash\left(L_{25} \cup L_{34} \cup L_{3}\right)\right) .
\end{aligned}
$$

These are cylinders isomorphic to $\mathbb{A}_{* *}^{1} \times \mathbb{A}^{1}$ (Example 2.6).

For a rational number $\varepsilon$ we have $-K_{\mathbb{P}^{2}} \sim_{\mathbb{Q}}(1-2 \varepsilon) L_{1}+(1+\varepsilon) L_{23}+(1+\varepsilon) L_{45}$. Therefore,

$$
-K_{S} \sim_{\mathbb{Q}}(1-2 \varepsilon) \widetilde{L}_{1}+(1+\varepsilon) \widetilde{L}_{23}+(1+\varepsilon) \widetilde{L}_{45}-2 \varepsilon E_{1}+\varepsilon \sum_{i=2}^{5} E_{i} .
$$

Thus we have

$$
A \sim_{\mathbb{Q}} \frac{1}{\mu}\left((1-2 \varepsilon) \widetilde{L}_{1}+(1+\varepsilon) \widetilde{L}_{23}+(1+\varepsilon) \widetilde{L}_{45}+\left(a_{1}-2 \varepsilon\right) E_{1}+\sum_{i=2}^{5}\left(a_{i}+\varepsilon\right) E_{i}\right)
$$

By taking $0<\varepsilon<a_{1} / 2$ we see that $U_{1}$ is an $A$-polar cylinder since

$$
U_{1}=S \backslash\left(\widetilde{L}_{23} \cup \widetilde{L}_{45} \cup \widetilde{L}_{1} \cup E_{1} \cup E_{2} \cup E_{3} \cup E_{4} \cup E_{5}\right) .
$$


Similarly $U_{2}$ and $U_{3}$ are also $A$-polar cylinders.

Let $C$ be the conic passing through the points $P_{1}, \ldots, P_{5}$ and take an arbitrary line $L$ tangent to the conic $C$. Consider the set

$$
U=\pi^{-1}\left(\mathbb{P}^{2} \backslash(C \cup L)\right),
$$

which is a cylinder isomorphic to $\mathbb{A}_{*}^{1} \times \mathbb{A}^{1}$.

For a rational number $\varepsilon$ we have $-K_{\mathbb{P}^{2}} \sim_{\mathbb{Q}}(1-2 \varepsilon) L+(1+\varepsilon) C$. Therefore,

$$
-K_{S} \sim_{\mathbb{Q}}(1-2 \varepsilon) \widetilde{L}+(1+\varepsilon) \widetilde{C}+\varepsilon \sum_{i=1}^{5} E_{i},
$$

where $\widetilde{L}$ and $\widetilde{C}$ are the proper transforms of $L$ and $C$ on $S$. Thus we have

$$
A \sim \mathbb{Q} \frac{1}{\mu}\left((1-2 \varepsilon) \widetilde{L}+(1+\varepsilon) \widetilde{C}+\sum_{i=1}^{5}\left(a_{i}+\varepsilon\right) E_{i}\right)
$$

By taking $0<\varepsilon<1 / 2$ we see that $U$ is an $A$-polar cylinder since

$$
U=S \backslash\left(\widetilde{L} \cup \widetilde{C} \cup E_{1} \cup E_{2} \cup E_{3} \cup E_{4} \cup E_{5}\right) .
$$

Note that

$$
U \cup U_{1} \cup U_{2} \cup U_{3}=S \backslash\left(E_{1} \cup E_{2} \cup E_{3} \cup E_{4} \cup E_{5}\right) .
$$

If the lines $L_{1}, L_{2}, L_{3}$ are distinct, then the three cylinders $U_{1}, U_{2}, U_{3}$ can cover $S \backslash\left(E_{1} \cup E_{2} \cup E_{3} \cup E_{4} \cup E_{5}\right)$ without the cylinder $U$.

Now consider different blow-downs of $S$ that send some $E_{i}$ onto a line in $\mathbb{P}^{2}$. Note that for the pair of the disjoint $(-1)$-curves $E_{i}$ and $E_{j}$, there is a unique $(-1)$-curve $E_{i j}$ that intersects $E_{i}$ and $E_{j}$ but none of the other exceptional curves of $\pi$. This is the proper transform of the line joining $P_{i}$ and $P_{j}$ by $\pi$.

Let $\{i, j, k\}$ be a subset of three elements in $\{1,2,3,4,5\}$ and let $\{\alpha, \beta\}$ be its complement. Let $\pi_{i j k}: S \rightarrow \mathbb{P}^{2}$ be the contraction of the five mutually disjoint $(-1)$ curves $E_{i j}, E_{j k}, E_{i k}, E_{\alpha}, E_{\beta}$. Then the images of the (-1)-curves $E_{i}, E_{j}, E_{k}$ by $\pi_{i j k}$ are lines on $\mathbb{P}^{2}$ and

$$
\begin{gathered}
\pi_{i j k}\left(E_{i}\right) \cap \pi_{i j k}\left(E_{j}\right)=\pi_{i j k}\left(E_{i j}\right), \quad \pi_{i j k}\left(E_{i}\right) \cap \pi_{i j k}\left(E_{k}\right)=\pi_{i j k}\left(E_{i k}\right), \\
\pi_{i j k}\left(E_{j}\right) \cap \pi_{i j k}\left(E_{k}\right)=\pi_{i j k}\left(E_{j k}\right) .
\end{gathered}
$$

There is a unique conic $C_{i j k}$ passing through the points $\pi_{i j k}\left(E_{i j}\right), \pi_{i j k}\left(E_{i k}\right)$, $\pi_{i j k}\left(E_{j k}\right), \pi_{i j k}\left(E_{\alpha}\right), \pi_{i j k}\left(E_{\beta}\right)$. Now assume that $a_{i} \geq a_{j} \geq a_{k}$ and take the line $L_{i j k} \subset \mathbb{P}^{2}$ tangent to the conic $C_{i j k}$ at the point $\pi_{i j k}\left(E_{i j}\right)$. Put

$$
V_{i j k}=\pi_{i j k}^{-1}\left(\mathbb{P}^{2} \backslash\left(C_{i j k} \cup L_{i j k}\right)\right) \text {. }
$$


This is a cylinder isomorphic to $\mathbb{A}_{*}^{1} \times \mathbb{A}^{1}$ (Example 2.7).

For an arbitrary rational number $\varepsilon$

$$
\begin{aligned}
-K_{\mathbb{P}^{2}} \sim_{\mathbb{Q}}\left(1+a_{i}+a_{k}+\varepsilon\right) C_{i j k} & +\left(1-a_{i}-a_{k}+a_{j}-2 \varepsilon\right) L_{i j k} \\
& -a_{i}\left(\pi_{i j k}\left(E_{i}\right)\right)-a_{j}\left(\pi_{i j k}\left(E_{j}\right)\right)-a_{k}\left(\pi_{i j k}\left(E_{k}\right)\right),
\end{aligned}
$$

and hence

$$
\begin{aligned}
-K_{S} \sim_{\mathbb{Q}}\left(1+a_{i}+a_{k}+\varepsilon\right) \widetilde{C}_{i j k} & +\left(1-a_{i}-a_{k}+a_{j}-2 \varepsilon\right) \widetilde{L}_{i j k} \\
& +\left(1-a_{i}-\varepsilon\right) E_{i j}+\left(a_{i}-a_{j}+\varepsilon\right) E_{j k}+\varepsilon E_{i k} \\
& +\left(a_{i}+a_{k}+\varepsilon\right) E_{\alpha}+\left(a_{i}+a_{k}+\varepsilon\right) E_{\beta} \\
& -a_{i} E_{i}-a_{j} E_{j}-a_{k} E_{k},
\end{aligned}
$$

where $\widetilde{C}_{i j k}$ and $\widetilde{L}_{i j k}$ are the proper transforms of $C_{i j k}$ and $L_{i j k}$ by the morphism $\pi_{i j k}$. Thus we have

$$
\begin{aligned}
\mu A \sim_{\mathbb{Q}}\left(1+a_{i}+a_{k}+\varepsilon\right) \widetilde{C}_{i j k} & +\left(1-a_{i}-a_{k}+a_{j}-2 \varepsilon\right) \widetilde{L}_{i j k} \\
& +\left(1-a_{i}-\varepsilon\right) E_{i j}+\left(a_{i}-a_{j}+\varepsilon\right) E_{j k}+\varepsilon E_{i k} \\
& +\left(a_{i}+a_{k}+a_{\alpha}+\varepsilon\right) E_{\alpha}+\left(a_{i}+a_{k}+a_{\beta}+\varepsilon\right) E_{\beta} .
\end{aligned}
$$

Since $a_{i}<1$, all coefficients in the divisor above are positive for a sufficiently small positive rational number $\varepsilon$. This shows that $V_{i j k}$ is an $A$-polar cylinder since

$$
S \backslash\left(\widetilde{C}_{i j k} \cup \widetilde{L}_{i j k} \cup E_{i j} \cup E_{j k} \cup E_{i k} \cup E_{\alpha} \cup E_{\beta}\right) \cong \mathbb{P}^{2} \backslash\left(C_{i j k} \cup L_{i j k}\right) .
$$

Since $E_{i} \subset V_{i j k} \cup V_{i \alpha \beta}$, we have

$$
E_{1} \cup E_{2} \cup E_{3} \cup E_{4} \cup E_{5} \subset \bigcup_{i, j, k=1}^{5} V_{i j k} .
$$

Consequently, we have obtained a covering of $S$ by $A$-polar cylinders:

$$
S=U \cup \bigcup_{\ell=1}^{3} U_{\ell} \cup \bigcup_{i, j, k=1}^{5} V_{i j k} .
$$

Now we suppose that there is a non-empty proper subset $W \subset S$ invariant with respect to all cylinders above.

Since the complement $S \backslash W$ is also a non-empty proper subset invariant with respect to all cylinders, up to switching the invariant sets $W$ and $S \backslash W$, we may assume that a point $w$ of $W$ is contained in $U_{\ell}$ for some $\ell$. Then $W$ must contain the fiber $F_{w}$ of $U_{\ell}$ passing through $w$. Without loss of generality, we may assume that $\ell=1$. Then the line $L=\overline{\pi\left(F_{w}\right)} \subset \mathbb{P}^{2}$ passes through the point $Q_{1}$. Since $Q_{1}, Q_{2}$ and $Q_{3}$ are not colinear, we may assume that $L$ does not pass through $Q_{2}$. Thus all $\mathbb{A}^{1}$-fibers of the 
cylinder $U_{2}$ intersect $L$, so that all of them should be contained in $W{ }^{1}$ Therefore, the complement $S \backslash W$ is contained in the support of finitely many curves.

No point of $S \backslash W$ is contained in $U_{1} \cup U_{2} \cup U_{3}$ : otherwise, the set $W$, the complement of $S \backslash W$, would be also contained in the support of finitely many curves for the same reason. Therefore, a point $w^{\prime}$ of $S \backslash W$ is contained either in $V_{i j k}$ for some subset $\{i, j, k\}$ of three elements in $\{1,2,3,4,5\}$ or in $U$. Either $V_{i j k}$ or $U$ has a fiber $F_{w^{\prime}}$ passing through the point $w^{\prime}$. If the fiber $F_{w^{\prime}}$ belongs to $V_{i j k}$, then $\overline{\pi_{i j k}\left(F_{w^{\prime}}\right)}$ is a conic passing through $\pi_{i j k}\left(w^{\prime}\right)$ and tangent to $L_{i j k}$ at $\pi_{i j k}\left(E_{i j}\right)$. If the fiber $F_{w^{\prime}}$ belongs to $U$, then $\overline{\pi\left(F_{w^{\prime}}\right)}$ is a conic passing through $\pi\left(w^{\prime}\right)$ and tangent to $L$. These show that $S \backslash W$ and $U_{1} \cup U_{2} \cup U_{3}$ have a common point. This is a contradiction.

Consequently, the covering of $S$ above is transversal. Therefore, the affine cone Affcone $_{A}(S)$ is flexible by Theorem 2.5.

\subsection{Conic bundle case}

Suppose that the contraction $\phi: S \rightarrow Z$ given by the face $\Delta_{(S, A)}$ is a conic bundle, i.e., $Z=\mathbb{P}^{1}$. The face $\Delta_{(S, A)}$ is spanned by an irreducible fiber $B$ of $\phi$ and four disjoint $(-1)$-curves $E_{1}, E_{2}, E_{3}, E_{4}$. We may then write

$$
K_{S}+\mu A \sim_{\mathbb{Q}} a B+\sum_{i=1}^{4} a_{i} E_{i}
$$

where $a$ is a positive rational number and $a_{i}$ are non-negative rational numbers. Let $\phi_{1}: S \rightarrow R$ be the birational morphism obtained by contracting the disjoint $(-1)$ curves $E_{1}, \ldots, E_{4}$.

Subcase 1: $R$ is isomorphic to the Hirzebruch surface $\mathbb{F}_{1}$.

In this subcase, we have an extra $(-1)$-curve $E_{5}$ which intersects the 0 -curve $B$ but none of $E_{1}, \ldots, E_{4}$. Contracting $\phi_{1}\left(E_{5}\right)$, the negative section of $\mathbb{F}_{1}$, we obtain a birational morphism $\phi_{2}: R \rightarrow \mathbb{P}^{2}$. Put $\pi=\phi_{2} \circ \phi_{1}: S \rightarrow \mathbb{P}^{2}$ and $P_{i}=\pi\left(E_{i}\right)$.

Let $C$ be a cuspidal cubic, with a cusp at $P_{5}$, that passes through $P_{1}, \ldots, P_{4}$. Let $T$ be the Zariski tangent line to $C$ at $P_{5}$. Put

$$
U_{C}=\pi^{-1}\left(\mathbb{P}^{2} \backslash(C \cup T)\right)
$$

It is a cylinder isomorphic to $\mathbb{A}_{*}^{1} \times \mathbb{A}^{1}$ (Example 2.8).

From $-K_{\mathbb{P}^{2}} \sim_{\mathbb{Q}}(1+\varepsilon) C-3 \varepsilon T$ with an arbitrary rational number $\varepsilon$, we obtain

$$
-K_{S} \sim_{\mathbb{Q}}(1+\varepsilon) \widetilde{C}-3 \varepsilon \widetilde{T}+\varepsilon \sum_{i=1}^{4} E_{i}+(1-\varepsilon) E_{5},
$$

\footnotetext{
1 This idea originates from [11, Subsection 3.1]
} 
where $\widetilde{C}$ and $\widetilde{T}$ are the proper transforms of $C$ and $T$, respectively. Since $B$ is linearly equivalent to $\widetilde{T}$,

$$
A \sim_{\mathbb{Q}} \frac{1}{\mu}\left((1+\varepsilon) \widetilde{C}+(a-3 \varepsilon) \widetilde{T}+\sum_{i=1}^{4}\left(a_{i}+\varepsilon\right) E_{i}+(1-\varepsilon) E_{5}\right)
$$

By taking a sufficiently small positive rational number $\varepsilon$, we see that the cylinder $U_{C}$ is $A$-polar because

$$
U_{C}=S \backslash\left(\widetilde{C} \cup \widetilde{T} \cup E_{1} \cup \cdots \cup E_{5}\right) .
$$

Let $\mathcal{B}$ be the collection of cuspidal cubics that pass through $P_{1}, \ldots, P_{5}$ and whose cusps are located at $P_{5}$. Then the cylinders $U_{C}, C \in \mathcal{B}$, cover $S$ except the exceptional curves $E_{1}, \ldots, E_{5}$.

For each (-1)-curve $E_{i}, i=1,2,3,4$, we have another $(-1)$-curve $E_{i}^{\prime}$ in the fiber of $\phi$ that contains $E_{i}$. It intersects both $E_{i}$ and $E_{5}$. The curve $B$ is of course linearly equivalent to the divisor $E_{i}+E_{i}^{\prime}$.

Let $\{\alpha, \beta, \gamma\}$ be the complement of the subset $\{i\}$ in $\{1,2,3,4\}$. Let $\pi_{i}: S \rightarrow \mathbb{P}^{2}$ be the contraction of the five mutually disjoint (-1)-curves $E_{\alpha \beta}, E_{\beta \gamma}, E_{\alpha \gamma}, E_{i}, E_{5}$, where $E_{\alpha \beta}$ is a unique (-1)-curve intersecting $E_{\alpha}, E_{\beta}$ but none of $E_{\gamma}, E_{i}, E_{5}$, and $E_{\beta \gamma}, E_{\alpha \gamma}$ are defined in the same manner.

The images of the (-1)-curves $E_{\alpha}, E_{\beta}, E_{\gamma}$ by $\pi_{i}$ are lines on $\mathbb{P}^{2}$ and

$$
\begin{gathered}
\pi_{i}\left(E_{\alpha}\right) \cap \pi_{i}\left(E_{\beta}\right)=\pi_{i}\left(E_{\alpha \beta}\right), \quad \pi_{i}\left(E_{\beta}\right) \cap \pi_{i}\left(E_{\gamma}\right)=\pi_{i}\left(E_{\beta \gamma}\right), \\
\pi_{i}\left(E_{\alpha}\right) \cap \pi_{i}\left(E_{\gamma}\right)=\pi_{i}\left(E_{\alpha \gamma}\right) .
\end{gathered}
$$

Moreover $\pi_{i}\left(E_{i}^{\prime}\right)$ is the conic in $\mathbb{P}^{2}$ passing through the points $\pi_{i}\left(E_{\alpha \beta}\right), \pi_{i}\left(E_{\beta \gamma}\right)$, $\pi_{i}\left(E_{\alpha \gamma}\right), \pi_{i}\left(E_{i}\right)$ and $\pi_{i}\left(E_{5}\right)$. Without loss of generality we may assume that $a_{\alpha} \geq$ $a_{\beta} \geq a_{\gamma}$. We then take the line $L_{i} \subset \mathbb{P}^{2}$ tangent to the conic $\pi_{i}\left(E_{i}^{\prime}\right)$ at the point $\pi_{i}\left(E_{\alpha \beta}\right)$. Put

$$
V_{i}=\pi_{i}^{-1}\left(\mathbb{P}^{2} \backslash\left(\pi_{i}\left(E_{i}^{\prime}\right) \cup L_{i}\right)\right)
$$

Then $V_{i}$ is a cylinder on $S$ because $\mathbb{P}^{2} \backslash\left(\pi_{i}\left(E_{i}^{\prime}\right) \cup L_{i}\right) \simeq \mathbb{A}_{*}^{1} \times \mathbb{A}^{1}$ (Example 2.7).

As in the birational morphism case, from

$$
\begin{aligned}
-K_{\mathbb{P}^{2}} \sim_{\mathbb{Q}}\left(1+a_{\alpha}+a_{\gamma}+\varepsilon\right)\left(\pi_{i}\left(E_{i}^{\prime}\right)\right) & +\left(1-a_{\alpha}-a_{\gamma}+a_{\beta}-2 \varepsilon\right) L_{i} \\
& -a_{\alpha}\left(\pi_{i}\left(E_{\alpha}\right)\right)-a_{\beta}\left(\pi_{i}\left(E_{\beta}\right)\right)-a_{\gamma}\left(\pi_{i}\left(E_{\gamma}\right)\right)
\end{aligned}
$$


with a sufficiently small positive rational number $\varepsilon$, we obtain

$$
\begin{aligned}
\mu A \sim_{\mathbb{Q}}\left(1+a_{\alpha}+a_{\gamma}+a+\varepsilon\right) E_{i}^{\prime} & +\left(1-a_{\alpha}-a_{\gamma}+a_{\beta}-2 \varepsilon\right) \widetilde{L}_{i} \\
& +\left(1-a_{\alpha}-\varepsilon\right) E_{\alpha \beta}+\left(a_{\alpha}-a_{\beta}+\varepsilon\right) E_{\beta \gamma} \\
& +\varepsilon E_{\alpha \gamma}+\left(a_{\alpha}+a_{\gamma}+a+a_{i}+\varepsilon\right) E_{i} \\
& +\left(a_{\alpha}+a_{\gamma}+\varepsilon\right) E_{5}
\end{aligned}
$$

where $\widetilde{L}_{i}$ are the proper transforms of $L_{i}$. This shows that $V_{i}$ is an $A$-polar cylinder because

$$
V_{i}=S \backslash\left(E_{i}^{\prime} \cup \widetilde{L}_{i} \cup E_{\alpha \beta} \cup E_{\beta \gamma} \cup E_{\alpha \gamma} \cup E_{i} \cup E_{5}\right)
$$

Moreover, the four cylinders $V_{1}, \ldots, V_{4}$ cover the (-1)-curves $E_{1}, E_{2}, E_{3}$ and $E_{4}$.

Let $\{i, j\}$ be a subset of two elements in $\{1,2,3,4\}$ and let $\{\alpha, \beta\}$ be its complement. For a pair of the disjoint (-1)-curves $E_{i}$ and $E_{j}$, there is a unique (-1)-curve $E_{i j}$ that intersects $E_{i}$ and $E_{j}$ but none of the other exceptional curves of $\pi$. As in the birational morphism case, $E_{i j}$ is the proper transform of the line joining $P_{i}$ and $P_{j}$ by $\pi$.

Let $\pi_{i j}: S \rightarrow \mathbb{P}^{2}$ be the contraction of the five mutually disjoint $(-1)$-curves $E_{i j}, E_{i 5}, E_{j 5}, E_{\alpha}, E_{\beta}$. Then the images of the (-1)-curves $E_{i}, E_{j}, E_{5}$ by $\pi_{i j}$ are lines on $\mathbb{P}^{2}$ and

$$
\begin{gathered}
\pi_{i j}\left(E_{i}\right) \cap \pi_{i j}\left(E_{j}\right)=\pi_{i j}\left(E_{i j}\right), \quad \pi_{i j}\left(E_{i}\right) \cap \pi_{i j}\left(E_{5}\right)=\pi_{i 5}\left(E_{i 5}\right), \\
\pi_{i j}\left(E_{j}\right) \cap \pi_{i j}\left(E_{5}\right)=\pi_{i j}\left(E_{j 5}\right) .
\end{gathered}
$$

The image of $B$ by $\pi_{i j}$ is a line passing through the point $\pi_{i j}\left(E_{i j}\right)$.

Take the conic $C_{i j}$ passing through the five points $\pi_{i j}\left(E_{i j}\right), \pi_{i j}\left(E_{i 5}\right), \pi_{i j}\left(E_{j 5}\right)$, $\pi_{i j}\left(E_{\alpha}\right), \pi_{i j}\left(E_{\beta}\right)$. Let $L_{i j}$ be the tangent line to the conic $C_{i j}$ at the point $\pi_{i j}\left(E_{i j}\right)$. Put

$$
V_{i j}=\pi_{i j}^{-1}\left(\mathbb{P}^{2} \backslash\left(C_{i j} \cup L_{i j}\right)\right)
$$

It is isomorphic to $\mathbb{A}_{*}^{1} \times \mathbb{A}^{1}$ (Example 2.7).

The union of $V_{i j}$ covers the $(-1)$-curves $E_{1}, \ldots, E_{5}$ except the intersection points of $E_{i}$ and $E_{i 5}, i=1,2,3,4$. In particular, it covers $E_{5}$ completely.

To show that $V_{i j}$ is $A$-polar, we may assume $a_{i} \geq a_{j}$ without loss of generality. For an arbitrary positive rational number $\varepsilon$

$$
-K_{\mathbb{P}^{2}} \sim_{\mathbb{Q}}\left(1+a_{i}+\varepsilon\right) C_{i j}+\left(1-a_{i}+a_{j}-2 \varepsilon\right) L_{i j}-a_{i}\left(\pi_{i j}\left(E_{i}\right)\right)-a_{j}\left(\pi_{i j}\left(E_{j}\right)\right) .
$$

Hence

$$
\begin{aligned}
-K_{S} \sim_{\mathbb{Q}}\left(1+a_{i}+\varepsilon\right) \widetilde{C}_{i j} & +\left(1-a_{i}+a_{j}-2 \varepsilon\right) \widetilde{L}_{i j} \\
& +\left(1-a_{i}-\varepsilon\right) E_{i j}+\left(a_{i}-a_{j}+\varepsilon\right) E_{j 5}+\varepsilon E_{i 5} \\
& +\left(a_{i}+\varepsilon\right) E_{\alpha}+\left(a_{i}+\varepsilon\right) E_{\beta}-a_{i} E_{i}-a_{j} E_{j}
\end{aligned}
$$


where $\widetilde{C}_{i j}$ and $\widetilde{L}_{i j}$ are the proper transforms of $C_{i j}$ and $L_{i j}$. Since $\widetilde{L}_{i j}$ is linearly equivalent to $B$, we have

$$
\begin{aligned}
\mu A \sim_{\mathbb{Q}}\left(1+a_{i}+\varepsilon\right) \widetilde{C}_{i j} & +\left(1-a_{i}+a_{j}+a-2 \varepsilon\right) \widetilde{L}_{i j} \\
& +\left(1-a_{i}-\varepsilon\right) E_{i j}+\left(a_{i}-a_{j}+\varepsilon\right) E_{j 5}+\varepsilon E_{i 5} \\
& +\left(a_{i}+a_{\alpha}+\varepsilon\right) E_{\alpha}+\left(a_{i}+a_{\beta}+\varepsilon\right) E_{\beta} .
\end{aligned}
$$

Since $a_{i}<1$, all coefficients in the divisor above are positive for a sufficiently small positive rational number $\varepsilon$. This implies that $V_{i j}$ is an $A$-polar cylinder because

$$
V_{i j}=S \backslash\left(\widetilde{C}_{i j} \cup \widetilde{L}_{i j} \cup E_{i j} \cup E_{j 5} \cup E_{i 5} \cup E_{\alpha} \cup E_{\beta}\right) .
$$

Consequently, we have constructed a covering of $S$ by $A$-polar cylinders:

$$
S=\bigcup_{C \in \mathcal{B}} U_{C} \cup \bigcup_{i=1}^{4} V_{i} \cup \bigcup_{i, j=1}^{4} V_{i j}
$$

Now we claim that the covering above is transversal. Suppose that this is not true. Then there is a non-empty proper subset $W$ of $S$ invariant to all cylinders in the covering. Let $w$ be an arbitrary point in $W$. The image $\pi(W)$ is invariant to the cylinder $\pi\left(U_{C}\right)$ for every $C \in \mathcal{B}$.

Since the complement $S \backslash W$ is also a non-empty proper subset invariant with respect to all cylinders, up to switching the invariant sets $W$ and $S \backslash W$, we may assume that $w$ lies in the cylinder $U_{C}$ for some $C \in \mathcal{B}$. There is a cuspidal cubic $C_{w}$ on $\mathbb{P}^{2}$ that becomes the $\mathbb{A}^{1}$-fiber of $\pi\left(U_{C}\right)$ passing through the point $\pi(w)$. This is a cuspidal cubic, passing through the point $\pi(w)$ with a cusp at $P_{5}$, whose Zariski tangent line at $P_{5}$ coincides with that of $C$. Then the affine curve $C_{w} \backslash\left\{P_{5}\right\}$ is contained in $\pi(W)$. Choose a cuspidal cubic $C^{\prime}$ in $\mathcal{B}$ whose Zariski tangent line at $P_{5}$ is different from that of $C$. Then the corresponding cylinder $U_{C^{\prime}}$ contains all points on $C_{w}$ except finitely many points. This implies that $S \backslash W$ is contained in the support of finitely many curves.

A point $w^{\prime}$ in $S \backslash W$ must lie either in $V_{i j}$ for some $i, j$ or in $V_{i}$ for some $i$ : otherwise $W$, the complement of $S \backslash W$, would be contained in the support of finitely many curves for the same reason. The cylinder $V_{i j}$ is given by the conic $C_{i j}$ passing through the five points $\pi_{i j}\left(E_{i j}\right), \pi_{i j}\left(E_{i 5}\right), \pi_{i j}\left(E_{j 5}\right), \pi_{i j}\left(E_{\alpha}\right), \pi_{i j}\left(E_{\beta}\right)$ and the tangent line $L_{i j}$ to the conic $C_{i j}$ at the point $\pi_{i j}\left(E_{i j}\right)$. The cylinder $V_{i}$ is defined by the conic $\pi_{k}\left(E_{i}^{\prime}\right)$ and the line $L_{i}$ tangent to the conic $\pi_{k}\left(E_{i}^{\prime}\right)$ at the point $\pi_{i}\left(E_{\alpha \beta}\right)$. Therefore, there is either a conic $F_{w^{\prime}}$ that passes through the point $\pi_{i j}\left(w^{\prime}\right)$ and tangent to the line $L_{i j}$ at the point $\pi_{i j}\left(E_{i j}\right)$ or a conic $G_{w^{\prime}}$ that passes through the point $\pi_{i}\left(w^{\prime}\right)$ and tangent to the line $L_{i}$ at the point $\pi_{i}\left(E_{\alpha \beta}\right)$. The former (resp. the latter) conic defines the $\mathbb{A}^{1}$-fiber of $V_{i j}$ (resp. $V_{i}$ ) passing through the point $w^{\prime}$. Therefore, $F_{w^{\prime}} \backslash\left\{\pi_{i j}\left(E_{i j}\right)\right\}$ (resp. $\left.G_{w^{\prime}} \backslash\left\{\pi_{i}\left(E_{\alpha \beta}\right)\right\}\right)$ is contained in $\pi_{i j}(S \backslash W)$ (resp. $\pi_{i}(S \backslash W)$ ). Since every cuspidal curve that defines an $\mathbb{A}^{1}$-fiber of $U_{C}$ intersects $F_{w^{\prime}}$ and $G_{w^{\prime}}$ at a point other than $\pi_{i j}\left(E_{i j}\right), \pi_{i}\left(E_{\alpha \beta}\right)$, and $P_{5}$, the set $W$, the complement of $S \backslash W$, is contained in the support of finitely many curves. This is a contradiction. 
Subcase 2: $R$ is isomorphic to $\mathbb{P}^{1} \times \mathbb{P}^{1}$.

The image $B$ by $\phi_{1}$ is a curve of bidegree $(1,0)$ or $(0,1)$ on $\mathbb{P}^{1} \times \mathbb{P}^{1}$. We may assume that $\phi_{1}(B)$ is a curve of bidegree $(0,1)$. There is a one-dimensional family $\mathcal{C}$ of irreducible curves of bidegree $(1,2)$ passing through the four points $\phi_{1}\left(E_{1}\right), \ldots, \phi_{1}\left(E_{4}\right)$. For each curve $C$ in the family $\mathcal{C}$, we take a curve $L_{C}$ of bidegree $(1,0)$ tangent to $C$ at a point. By $H_{C}$ denote the curve of bidegree $(0,1)$ that passes through the intersection point of $C$ and $L_{C}$. These three curves $C, L_{C}$ and $H_{C}$ define a cylinder isomorphic to an $\mathbb{A}^{1}$-bundle over $\mathbb{A}_{*}^{1}$ (Example 2.9). Put

$$
U_{C}=\phi_{1}^{-1}\left(\mathbb{P}^{1} \times \mathbb{P}^{1} \backslash\left(C \cup L_{C} \cup H_{C}\right)\right) .
$$

For an arbitrary rational number $\varepsilon$

$$
-K_{\mathbb{P}^{1} \times \mathbb{P}^{1}} \sim_{\mathbb{Q}}(1+\varepsilon) C+(1-\varepsilon) L_{C}+(a-2 \varepsilon) H_{C}-a \phi_{1}(B) .
$$

Now let $\delta_{i}$ be 1 if $L_{C}$ passes through $\phi\left(E_{i}\right)$ and 0 if not. Then we obtain

$$
\begin{aligned}
-K_{S} \sim_{\mathbb{Q}}(1+\varepsilon) \widetilde{C}+(1-\varepsilon) \widetilde{L}_{C} & +(a-2 \varepsilon) \widetilde{H}_{C}-a B \\
& +\sum_{i=1}^{4}\left(\varepsilon+(1+a-3 \varepsilon) \delta_{i}\right) E_{i},
\end{aligned}
$$

where $\widetilde{C}, \widetilde{L}_{C}$ and $\widetilde{H}_{C}$ are the proper transforms of $C, L_{C}$ and $H_{C}$, respectively. Thus we have

$$
\begin{aligned}
A \sim \mathbb{Q} \frac{1}{\mu}\left((1+\varepsilon) \widetilde{C}+(1-\varepsilon) \widetilde{L}_{C}\right. & +(a-2 \varepsilon) \widetilde{H}_{C} \\
& \left.+\sum_{i=1}^{4}\left(a_{i}+\varepsilon+(1+a-3 \varepsilon) \delta_{i}\right) E_{i}\right) .
\end{aligned}
$$

By taking a sufficiently small positive rational number $\varepsilon$, we can see that $U_{C}$ is an A-polar cylinder since

$$
U_{C}=S \backslash\left(\widetilde{C} \cup \widetilde{L}_{C} \cup \widetilde{H}_{C} \cup E_{1} \cup E_{2} \cup E_{3} \cup E_{4}\right) .
$$

The cylinders defined by $C$ in $\mathcal{C}$ in this manner cover $S$ except $E_{1}, E_{2}, E_{3}, E_{4}$.

For each $(-1)$-curve $E_{i}$, we have another $(-1)$-curve $E_{i}^{\prime}$ in the fiber of $\phi$ that contains $E_{i}$. In addition, there is a $(-1)$-curve $E_{i}^{\prime \prime}$ that intersects $E_{i}$ but none of the other exceptional curves of $\phi_{1}$. The curve $B$ is linearly equivalent to the divisor $E_{i}+E_{i}^{\prime}$.

Let $\{\alpha, \beta, \gamma\}$ be the complement of the subset $\{i\}$ in $\{1,2,3,4\}$. The $(-1)$-curves $E_{\alpha}, E_{\beta}, E_{\gamma}, E_{i}^{\prime}, E_{i}^{\prime \prime}$ are mutually disjoint. Let $\pi_{i}: S \rightarrow \mathbb{P}^{2}$ be the contraction of the five mutually disjoint (-1)-curves $E_{\alpha \beta}, E_{\beta \gamma}, E_{\alpha \gamma}, E_{i}^{\prime}, E_{i}^{\prime \prime}$, where $E_{\alpha \beta}$ is a unique $(-1)$-curve intersecting $E_{\alpha}, E_{\beta}$ but none of $E_{\gamma}, E_{i}^{\prime}, E_{i}^{\prime \prime}$, and $E_{\beta \gamma}, E_{\alpha \gamma}$ are defined in the same manner. 
The images of the $(-1)$-curves $E_{\alpha}, E_{\beta}, E_{\gamma}$ by $\pi_{i}$ are lines on $\mathbb{P}^{2}$ and

$$
\begin{gathered}
\pi_{i}\left(E_{\alpha}\right) \cap \pi_{i}\left(E_{\beta}\right)=\pi_{i}\left(E_{\alpha \beta}\right), \quad \pi_{i}\left(E_{\beta}\right) \cap \pi_{i}\left(E_{\gamma}\right)=\pi_{i}\left(E_{\beta \gamma}\right), \\
\pi_{i}\left(E_{\alpha}\right) \cap \pi_{i}\left(E_{\gamma}\right)=\pi_{i}\left(E_{\alpha \gamma}\right) .
\end{gathered}
$$

Moreover $\pi_{i}\left(E_{i}\right)$ is the conic in $\mathbb{P}^{2}$ passing through the points $\pi_{i}\left(E_{\alpha \beta}\right), \pi_{i}\left(E_{\beta \gamma}\right)$, $\pi_{i}\left(E_{\alpha \gamma}\right), \pi_{i}\left(E_{i}^{\prime}\right)$ and $\pi_{i}\left(E_{i}^{\prime \prime}\right)$. Without loss of generality we may assume that $a_{\alpha} \geq$ $a_{\beta} \geq a_{\gamma}$. We then take the line $L_{i} \subset \mathbb{P}^{2}$ tangent to the conic $\pi_{i}\left(E_{i}\right)$ at the point $\pi_{i}\left(E_{\alpha \beta}\right)$. Put

$$
V_{i}=\pi_{i}^{-1}\left(\mathbb{P}^{2} \backslash\left(\pi_{i}\left(E_{i}\right) \cup L_{i}\right)\right)
$$

Then $V_{i}$ is a cylinder on $S$ because $\mathbb{P}^{2} \backslash\left(\pi_{i}\left(E_{i}\right) \cup L_{i}\right) \simeq \mathbb{A}_{*}^{1} \times \mathbb{A}^{1}$ (Example 2.7).

As before, for a sufficiently small positive rational number $\varepsilon$,

$$
\begin{aligned}
-K_{\mathbb{P}^{2}} \sim_{\mathbb{Q}}\left(1+a_{\alpha}+a_{\gamma}+\varepsilon\right)\left(\pi_{i}\left(E_{i}\right)\right) & +\left(1-a_{\alpha}-a_{\gamma}+a_{\beta}-2 \varepsilon\right) L_{i} \\
& -a_{\alpha}\left(\pi_{i}\left(E_{\alpha}\right)\right)-a_{\beta}\left(\pi_{i}\left(E_{\beta}\right)\right)-a_{\gamma}\left(\pi_{i}\left(E_{\gamma}\right)\right)
\end{aligned}
$$

yields an effective $\mathbb{Q}$-divisor

$$
\begin{aligned}
\mu A \sim_{\mathbb{Q}}\left(1+a_{\alpha}+a_{\gamma}+a+a_{i}+\varepsilon\right) E_{i} & +\left(1-a_{\alpha}-a_{\gamma}+a_{\beta}-2 \varepsilon\right) \widetilde{L}_{i} \\
& +\left(1-a_{\alpha}-\varepsilon\right) E_{\alpha \beta}+\left(a_{\alpha}-a_{\beta}+\varepsilon\right) E_{\beta \gamma} \\
& +\varepsilon E_{\alpha \gamma}+\left(a_{\alpha}+a_{\gamma}+a+\varepsilon\right) E_{i}^{\prime} \\
& +\left(a_{\alpha}+a_{\gamma}+\varepsilon\right) E_{i}^{\prime \prime}
\end{aligned}
$$

where $\widetilde{L}_{i}$ are the proper transforms of $L_{i}$. This shows that $V_{i}$ is an $A$-polar cylinder because

$$
V_{i}=S \backslash\left(E_{i} \cup \widetilde{L}_{i} \cup E_{\alpha \beta} \cup E_{\beta \gamma} \cup E_{\alpha \gamma} \cup E_{i}^{\prime} \cup E_{i}^{\prime \prime}\right)
$$

Moreover, the four cylinders $V_{i}$ cover the (-1)-curves $E_{1}, E_{2}, E_{3}$ and $E_{4}$.

We have obtained a covering of $S$ by $A$-polar cylinders:

$$
S=\bigcup_{C \in \mathcal{C}} U_{C} \cup \bigcup_{i=1}^{4} V_{i}
$$

Finally we claim that the covering above is transversal. Suppose that it is not transversal. Then there is a non-empty proper subset $W$ of $S$ that is invariant with respect to all cylinders in the covering above. Let $w$ be a point in $W$.

As in the previous cases, we may assume that $w$ belongs to $U_{C}$ for some $C \in \mathcal{C}$. The set $\phi_{1}(W) \subset \mathbb{P}^{1} \times \mathbb{P}^{1}$ is invariant to the cylinder $\phi_{1}\left(U_{C}\right)$. Let $P$ be the intersection point of $C$ and $L_{C}$. Then there is a curve $C_{w}$ of bidegree $(1,2)$ tangent to $L_{C}$ at the point $P$ and passing through the point $\phi_{1}(w)$. Then the affine curve $C_{w} \backslash\{P\}$ defines 
the $\mathbb{A}^{1}$-fiber in the cylinder $U_{C}$ passing through the point $w$. Therefore, $\phi_{1}^{-1}\left(C_{w} \backslash\{P\}\right)$ is contained in $W$. We can choose a curve $C^{\prime}$ in $\mathcal{C}$ that intersects $C_{w}$ at four distinct points. Therefore, every $\mathbb{A}^{1}$-fiber in $U_{C^{\prime}}$ except finitely many fibers must be contained in $W$. This shows that its complement $S \backslash W$ is contained in the support of finitely many curves.

A point in $S \backslash W$ cannot belong to the cylinder $U_{C}$ for any $C \in \mathcal{C}$ : otherwise $W$ would be contained in the support of finitely many curves. Therefore, a point $w^{\prime}$ in $S \backslash W$ is contained in $V_{i}$ for some $i$. Then there is a conic $C_{w^{\prime}}$ on $\mathbb{P}^{2}$ tangent to $L_{i}$ at the point $\pi_{i}\left(E_{\alpha \beta}\right)$ and passing through the point $\pi_{i}\left(w^{\prime}\right)$. This conic defines the $\mathbb{A}^{1}$-fiber of $V_{i}$ passing through the point $w^{\prime}$. Therefore, $C_{w^{\prime}} \backslash\left\{\pi_{i}\left(E_{\alpha \beta}\right)\right\}$ is contained in $\pi_{i}(S \backslash W)$. Since every curve of bidegree $(1,2)$ on $\mathbb{P}^{1} \times \mathbb{P}^{1}$ that defines an $\mathbb{A}^{1}$-fiber of $U_{C}$ intersects the curve $\phi_{1}\left(\pi_{i}^{-1}\left(C_{w^{\prime}}\right)\right)$ of bidegree $(3,3)$ outside $\phi_{1}\left(E_{\alpha \beta}\right)$, the set $W$, the complement of $S \backslash W$, is contained in the support of finitely many curves. This is a contradiction.

This completes the proof of Main Theorem.

\section{References}

1. Arzhantsev, I., Flenner, H., Kaliman, S., Kutzschebauch, F., Zaidenberg, M.: Flexible varieties and automorphism groups. Duke Math. J. 162(4), 767-823 (2013)

2. Arzhantsev, I., Flenner, H., Kaliman, S., Kutzschebauch, F., Zaidenberg, M.: Infinite transitivity on affine varieties. In: Bogomolov, F., Hassett, B., Tschinkel, Yu. (eds.) Birational Geometry, Rational Curves, and Arithmetic, pp. 1-13. Springer, New York (2013)

3. Arzhantsev, I.V., Kuyumzhiyan, K.G., Zaidenberg, M.G.: Flag varieties, toric varieties, and suspensions: three instances of infinite transitivity. Sb. Math. 203(7), 923-949 (2012)

4. Bogomolov, F., Karzhemanov, I., Kuyumzhiyan, K.: Unirationality and existence of infinitely transitive models. In: Bogomolov, F., Hassett, B., Tschinkel, Yu. (eds.) Birational Geometry, Rational Curves, and Arithmetic, pp. 77-92. Springer, New York (2013)

5. Cheltsov, I., Park, J., Won, J.: Affine cones over smooth cubic surfaces (2013). J. Eur. Math. Soc. (to appear)

6. Cheltsov, I., Park, J., Won, J.: Cylinders in singular del Pezzo surfaces (2013). arXiv:1311.5257

7. Dolgachev, I.V.: Classical Algebraic Geometry. Cambridge University Press, Cambridge (2012)

8. Kaliman, S., Zaidenberg, M.: Affine modification and affine hypersurfaces with a very transitive automorphism group. Transform. Groups 4(1), 53-95 (1999)

9. Kishimoto, T., Prokhorov, Yu., Zaidenberg, M.: $\mathbb{G}_{\mathrm{a}}$-actions on affine cones. Transform. Groups 18(4), $1137-1153$ (2013)

10. Kishimoto, T., Prokhorov, Yu., Zaidenberg, M.: Unipotent group actions on del Pezzo cones. Algebraic Geom. 1(1), 46-56 (2014)

11. Perepechko, A.Yu.: Flexibility of affine cones over del Pezzo surfaces of degree 4 and 5. Funct. Anal. Appl. 47(4), 284-289 (2013) 